\title{
Escolha da medicina como profissão e perspectiva laboral dos estudantes
}

\author{
The choice of Medicine as a profession and the students' labor perspective
}

\author{
Eduardo Delatorre Kamijo' (D) | eduardokamijo@hotmail.com \\ Maria Victória Schweder de Lima' (1) mariavictoriaschwederdelima@hotmail.com \\ Ana Paula Pereira' (D) ana.pereira@unoesc.edu.br \\ Elcio Luiz Bonamigo' ${ }^{1}$ (D)
}

\section{RESUMO}

Introdução: A medicina é uma profissão de prestígio, e, por isso, embora a concorrência para a admissão seja acirrada e os estudos exijam dedicação e sacrifícios, todos os anos milhares de jovens enfrentam o desafio de se tornarem médicos.

Objetivo: Este estudo teve como objetivo descrever as razões pelas quais os acadêmicos de um curso de Medicina escolheram a medicina como profissão e suas preocupações quanto à perspectiva laboral.

Método: Trata-se de uma pesquisa quantitativa, descritiva e documental, realizada por meio da aplicação de questionário com questões sociodemográficas e específicas.

Resultado: Destacou-se elevada percentagem do gênero feminino, com 63,31\% dos participantes. As principais razões de escolha da medicina como profissão foram altruísmo $(71,75 \%)$, estabilidade financeira $(59,42 \%)$ e realização pessoal $(58,77 \%)$. Entretanto, houve diminuição da preferência pelo altruísmo durante o internato $(p<0,01)$. A maior preocupação após a formação foi "realizar um bom trabalho/ser um bom profissional" (79,87\%), e, em relação ao "mercado de trabalho" e à "desvalorização da profissão", verificou-se aumento da preocupação durante o internato $(p<0,001)$. A maioria $(91,92 \%)$ manifestou a intenção de tornar-se médico especialista, sendo mais desejada a especialidade ginecologia e obstetrícia. Em relação à forma de trabalho, 51,3\% apontaram que gostariam de trabalhar como profissionais autônomos, 55,52\% manifestaram o desejo de ter um emprego público ou particular, e 7,92\% mencionaram a atuação como profissionais liberais. A maior dificuldade esperada na profissão foi a concorrência (57,70\%), e o requisito mais importante para o exercício profissional foi a "medicina como meio para ser útil ou ajudar pessoas" (98,38\%). Sobre os sentimentos experimentados como estudante, os níveis de ansiedade, estresse e sensação de sobrecarga foram elevados, respectivamente $80,52 \%, 79,55 \%$ e $73,38 \%$.

Conclusão: No início do curso, existe uma visão mais idealizada da medicina, e, no desenvolver das fases, conforme ocorre o contato com a prática médica, os acadêmicos percebem melhor as dificuldades e aumentam suas preocupações, sobretudo com o mercado de trabalho e a desvalorização da profissão, provocando, em alguns, a diminuição dos ideais humanísticos que motivaram a escolha da profissão, aspecto a ser valorizado pelas escolas de Medicina durante o ensino.

Palavras-chave: Medicina; Estudantes de Medicina; Escolha da Profissão; Altruísmo.

\section{ABSTRACT}

Introduction: Medicine is a prestigious profession, therefore, although competition for admission is fierce and studies require dedication and sacrifices, thousands of young people face the challenge of becoming doctors every year.

Objective: Describe the reasons why medical school students chose Medicine as a profession and their concerns about the job perspective.

Method: Quantitative descriptive and documentary research, through the application of a questionnaire with sociodemographic and specific questions.

Result: A high percentage of the female gender stood out, with $63.31 \%$ of the participants. The main reasons for choosing Medicine as a profession were altruism (71.75\%), financial stability (59.42\%) and personal accomplishment (58.77\%). However, there was a decrease in the preference for altruism during internship $(p<0.01)$. The biggest concern after training was "Doing a good job / Being a good professional" (79.87\%) and, in relation to the "job market" and "devaluation of the profession", there was an increase in the concern during internship ( $p<0.001)$. The majority (91.92\%) expressed the intention to become a specialist physician, with the specialty of Gynecology and Obstetrics being the most sought after. Regarding the way of working, $51.3 \%$ expressed the desire to be a self-employed professional, 55.52\% a public or private employee and $7.92 \%$ a liberal professional. The greatest difficulty expected in the profession was competition (57.70\%) and the most important requirement for professional practice was "Medicine as a means to be useful or help people" (98.38\%). Regarding the feelings experienced as a student, the levels of anxiety, stress and feeling of overload were high, respectively $80.52 \%, 79.55 \%$ and $73.38 \%$.

Conclusion: At the beginning of the course, there is a more idealized view of Medicine and throughout the development of phases, as contact with medical practice occurs, the students better understand their difficulties and increase their concerns, especially with the job market and the devaluation of the profession, causing, in some, the reduction of the humanistic ideals that motivated the choice of the profession, an aspect to be valued by medical schools during professional training.

Keywords: Medicine; Medical Students; Choice of Profession; Altruism.

1 Universidade do Oeste de Santa Catarina, Joaçaba, Santa Catarina, Brasil.

Editora-chefe: Rosiane Viana Zuza Diniz. | Editora associada: Izabel Cristina Meister Martins Coelho

Recebido em 09/03/21; Aceito em 28/09/21. | Avaliado pelo processo de double blind review. 


\section{INTRODUÇÃO}

A amenização das dores, a cura de doenças, a possibilidade de salvar vidas e a mobilização da própria vida em prol do outro eram os pilares que sustentavam as atividades médicas desde seu início, porém, com o passar dos anos e a propagação de outros ideais, essa ciência ficou mais ligada às questões econômicas em detrimento das humanitárias' $O$ reflexo desse crescente interesse financeiro alavancou a disseminação de novas faculdades de Medicina pelo território brasileiro e a permissão de abertura de mais vagas em faculdades já existentes que são adaptadas em locais muitas vezes sem estrutura adequada para a educação qualificada ${ }^{2}$.

Um total de 357 escolas médicas estavam em funcionamento em 2020, índice de 1,68 por milhão de habitantes, oferecendo 45.337 vagas com uma taxa de concorrência de 23,19, num país que já conta com mais de 500 mil médicos². Levando isso em conta, questiona-se sobre a qualidade da graduação médica com a formação de tantos novos profissionais, aspecto que aumenta a preocupação dos estudantes de Medicina.

Hipócrates recomendava aos médicos que fossem portadores de habilidades naturais, cultura, perseverança, dedicação e disposição para o estudo e o trabalho, aspectos que já prenunciavam as dificuldades da profissão ${ }^{3}$. Assim, o curso de Medicina é visto como um dos mais difíceis, por exigir intensa dedicação, sacrifícios e resistência física e emocional dos acadêmicos ${ }^{4}$. Entretanto, todos os anos, milhares de candidatos enfrentam verdadeiras batalhas nos vestibulares para serem médicos.

A graduação em Medicina abrange tanto os aspectos cognitivos e a dedicação ao estudo, quanto suscita questões emocionais intrínsecas à profissão ${ }^{5}$. $O$ estresse dos estudantes de Medicina é frequente e resulta de uma série de fatores, como extensa carga horária, falta de tempo para lazer e vida pessoal, competividade no curso e contato direto com o sofrimento que inclusive concorrem para a queda de sua qualidade de vida ${ }^{6}$. 0 surgimento do estresse é mais frequente no início do curso devido às dificuldades de adaptação ao ritmo universitário e às disciplinas cursadas em que predominam as atividades teórico-práticas ${ }^{7}$.

Um estudo com o intuito de levantar os fatores determinantes da escolha do curso de Medicina como profissão obteve frequência alta de respostas que mencionavam a facilidade de inserção no mercado de trabalho e os bons salários $^{8}$. Contudo, embora se mantenha elevado o prestígio da profissão, constata-se que há depreciação do trabalho na área da saúde e aumento da concorrência, o que tem despertado a preocupação dos estudantes ${ }^{9}$.

Sendo assim, estudos que permitam maior compreensão do motivo que leva um número tão grande de jovens a optar pela profissão médica e expliquem como evoluem os sentimentos deles ao longo do curso são essenciais para planejar e adequar sua formação. Para tanto, o presente trabalho teve como objetivos principais apontar as razões pelas quais os acadêmicos da primeira à $12^{\text {a }}$ fase de uma faculdade de Medicina escolheram esse curso como opção profissional e identificar suas preocupações quanto à perspectiva laboral.

\section{MÉTODO}

Quanto à natureza dos dados, a pesquisa é quantitativa e documental. Em relação aos objetivos, é descritiva. No que concerne às fontes de informação e aos procedimentos de coleta, trata-se de uma pesquisa documental. Participaram da amostra 308 estudantes da primeira à $12^{\text {a }}$ fase do curso de Medicina da Universidade do Oeste de Santa Catarina (Unoesc) - de um total de 372. No período de julho a novembro de 2018, esses estudantes responderam a sete questões que abordavam as razões que os levaram a escolher a medicina como profissão, as preocupações em relação ao futuro após formados, a maneira como desejavam exercer a profissão, as dificuldades que esperavam encontrar no exercício profissional e as características que consideravam importantes para a profissão médica. Aplicou-se ainda um questionário sociodemográfico. Os temas das perguntas foram organizados com base nas respostas de questionários diagnósticos aplicados a 226 estudantes da quarta e sétima fases, nas disciplinas de Ética e Sociedade (Bioética) e Ética Médica, entre 2013 e 2018. Os dados foram armazenados na planilha do Excel e posteriormente submetidos à análise estatística por meio dos testes exato de Fisher e qui-quadrado, sendo especialmente analisada a diferença da percepção dos estudantes nos ciclos pré-internato, cuja duração é de 3,5 anos, da primeira à sétima fase $(n=207$; $67,21 \%$ ) e internato, cuja duração é de 2,5 anos, da oitava a $12^{\mathrm{a}}$ fase $(n=101 ; 32,79 \%)$.

\section{RESULTADOS}

Em relação às características sociodemográficas, destacou-se a predominância do sexo feminino com 63,31\% (195) dos participantes, dos quais seis não marcaram nenhuma opção, mas não houve diferença significativa entre os grupos ( $p=0,4586)$. A faixa etária que respondeu ao maior número de questões foi de 22-26 anos, com 155 (50,32\%), seguida, respectivamente, dos grupos de 16-21 anos, com 128 (41,56\%), e de 27 anos ou mais, com 16 (5,19\%), dos quais nove não informaram a idade.

A maioria declarou ter renda familiar de quatro a dez salários mínimos ( $n=118 ; 38,31 \%$ ); outros indicaram renda de dez a 20 salários ( $n=103 ; 33,44 \%)$; e a minoria mencionou renda acima de 20 salários ( $n=44 ; 14,28 \%$ ). Na faixa de dois salários mínimos, havia 11 participantes (3,57\%), e de dois a quatro 
salários, 27 (8,77\%); cinco participantes não mencionaram a renda familiar. Não houve diferença significativa na distribuição das faixas de renda entre os grupos ( $p=0,1736)$.

Quando perguntados a respeito de qual a principal razão que os levou a escolher a medicina como futura profissão - nessa questão, os participantes poderiam marcar mais de uma opção -, obteve-se a seguinte ordem de frequência das respostas: "altruísmo" (71,75\%), "estabilidade financeira/bom salário/bom retorno financeiro" (59,42\%) e "realização pessoal" (58,77\%), conforme mostra a Tabela 1. Quando agrupadas as fases, houve diminuição significativa durante o internato das respostas "altruísmo" ( $p<0,01)$, "oportunidade de lidar ou ter contato com pessoas" $(p<0,01)$ e "curiosidade científica/busca por conhecimento humano" $(p<0,05)$.

Em relação ao futuro, depois de formado, a principal preocupação foi "realizar um bom trabalho/ser um bom profissional" (79,87\%), seguida de "passar na residência/obter qualificação"(64,29\%) e"medo de cometer erros/responsabilidades da profissão" (52,27\%), conforme mostra a Tabela 2.

Sobre a pós-graduação, 7,79\% manifestaram intenção de seguir como médicos generalistas não especialistas e $92,21 \%$ como médicos especialistas. Quando comparados os

Tabela 1. Razões da opção pelo curso de Medicina.

\begin{tabular}{|c|c|c|c|c|}
\hline \multirow{3}{*}{ Perguntas relacionadas à escolha da profissão } & Total & Pré-internato & Internato & \multirow{3}{*}{$\mathbf{P}$} \\
\hline & $\%(n)$ & $\%(n)$ & $\%(n)$ & \\
\hline & $100(308)$ & $67,21(207)$ & $32,79(101)$ & \\
\hline Altruísmo & $71,75(221)$ & $77,29(160)$ & $60,40(61)$ & ** \\
\hline Estabilidade financeira/bom salário/bom retorno financeiro & $59,42(183)$ & $60,39(125)$ & $57,43(58)$ & 0,6234 \\
\hline $\begin{array}{l}\text { Status/prestígio ou reconhecimento social do médico ou da } \\
\text { medicina }\end{array}$ & $4,55(14)$ & $5,31(11)$ & $2,97(3)$ & 0,5249 \\
\hline curiosidade científica/busca por conhecimento humano & $34,09(105)$ & $38,65(80)$ & $24,75(25)$ & * \\
\hline Oportunidade de lidar ou ter contato com pessoas & $36,36(112)$ & $41,55(86)$ & $25,74(26)$ & ** \\
\hline Influência familiar ou de terceiros, doença pessoal ou na família & $15,91(49)$ & $14,98(31)$ & $17,82(18)$ & 0,5120 \\
\hline Amor/admiração pela profissão ou por profissionais médicos & $51,95(160)$ & $55,56(115)$ & $44,55(45)$ & 0,0887 \\
\hline $\begin{array}{l}\text { Vocação/aptidão pessoal ou afinidade com a área da saúde ou } \\
\text { biológica }\end{array}$ & $54,22(167)$ & $57,49(119)$ & $47,52(48)$ & 0,1136 \\
\hline Realização pessoal & $58,77(181)$ & $58,94(122)$ & $58,42(59)$ & 1,00 \\
\hline Curso ou vestibular desafiador & $8,44(26)$ & $8,21(17)$ & $8,91(9)$ & 0,8297 \\
\hline
\end{tabular}

Os valores apresentam as frequências relativa e absoluta para as perguntas avaliadas. Utilizou-se o teste exato de Fisher. A diferença estatística entre os grupos pré-internato e internato foi considerada significativa quando ${ }^{*} p<0,05$ e $^{* *} p<0,01$.

Fonte: Elaborada pelos autores.

Tabela 2. Preocupações após formados.

\begin{tabular}{|c|c|c|c|c|}
\hline \multirow{3}{*}{$\begin{array}{l}\text { Perguntas relacionadas à preocupação com o futuro } \\
\text { depois de formados }\end{array}$} & Total & Pré-internato & Internato & \multirow{3}{*}{$\mathbf{P}$} \\
\hline & $\%(n)$ & $\%(n)$ & $\%(n)$ & \\
\hline & $100(308)$ & $67,21(207)$ & $32,79(101)$ & \\
\hline $\begin{array}{l}\text { Passar na residência/obter qualificação/ter conhecimento } \\
\text { suficiente }\end{array}$ & 64,29 (198) & $61,84(128)$ & $69,31(70)$ & 0,2081 \\
\hline Mercado de trabalho/desvalorização da profissão & $34,42(106)$ & $28,02(58)$ & $47,52(48)$ & *** \\
\hline Medo de cometer erros e das responsabilidades da profissão & $52,27(161)$ & $53,14(110)$ & $50,50(51)$ & 0,7159 \\
\hline Tempo para conciliar a carreira e a vida pessoal & $37,99(117)$ & $42,51(88)$ & $28,71(29)$ & * \\
\hline Infraestrutura precária para exercer a profissão & $17,86(55)$ & $18,36(38)$ & $16,83(17)$ & 0,8742 \\
\hline
\end{tabular}

Os valores apresentam as frequências relativa e absoluta para as perguntas avaliadas. Utilizou-se o teste exato de Fisher. A diferença estatística entre os grupos pré-internato e internato foi considerada significativa quando ${ }^{*} p<0,05$ e $^{* * *} p<0,001$.

Fonte: Elaborada pelos autores. 
ciclos, $89,86 \%$ dos estudantes do pré-internato e $97,03 \%$ dos estudantes do internato marcaram a opção de ser médico especialista $(p<0,05)$. Dentre as especialidades, a mais desejada foi ginecologia e obstetrícia $(6,17 \%)$, seguida de pediatria $(4,55 \%)$, cirurgia geral $(4,22 \%)$, psiquiatria $(3,57 \%)$ e oncologia $(3,25 \%)$, conforme mostra a Tabela 3.

Tabela 3. Escolha da especialidade.

\begin{tabular}{|c|c|c|c|c|c|}
\hline \multirow{2}{*}{\multicolumn{2}{|c|}{ Especialidades citadas }} & \multirow{3}{*}{$\begin{array}{c}\text { Total } \\
\%(n) \\
100(308)\end{array}$} & \multirow{3}{*}{$\begin{array}{c}\text { Pré-internato } \\
\%(n) \\
67,21(207) \\
\end{array}$} & \multirow{3}{*}{$\begin{array}{c}\text { Internato } \\
\%(n) \\
32,79(101) \\
\end{array}$} & \multirow{3}{*}{ p } \\
\hline & & & & & \\
\hline & & & & & \\
\hline Generalista & Não & $92,21(284)$ & $89,86(186)$ & $97,03(98)$ & \\
\hline \multirow[t]{26}{*}{ Especialista } & Não & $7,47(23)$ & $10,14(21)$ & $1,98(2)$ & ** \\
\hline & Pediatria & $4,55(14)$ & $3,86(8)$ & $5,94(6)$ & \\
\hline & Cirurgia geral & $4,22(13)$ & $2,90(6)$ & $6,93(7)$ & \\
\hline & Psiquiatria & $3,57(11)$ & $4,35(9)$ & $1,98(2)$ & \\
\hline & Oncologia & $3,25(10)$ & $3,38(7)$ & $2,97(3)$ & \\
\hline & Clínica médica & $1,95(6)$ & $0,48(1)$ & $4,95(5)$ & \\
\hline & Neurocirurgia & $1,62(5)$ & $1,93(4)$ & $0,99(1)$ & \\
\hline & Anestesiologia & $1,62(5)$ & $0,48(1)$ & $3,96(4)$ & \\
\hline & Dermatologia & $1,30(4)$ & $1,45(3)$ & $0,99(1)$ & \\
\hline & Cirurgia plástica & $1,30(4)$ & $1,93(4)$ & $0(0)$ & \\
\hline & Endocrinologia & $1,30(4)$ & $1,93(4)$ & $0(0)$ & \\
\hline & Cirurgia oncológica & $0,97(3)$ & $0(0)$ & $2,97(3)$ & \\
\hline & Geriatria & $0,97(3)$ & $1,45(3)$ & $0(0)$ & \\
\hline & Cirurgia do aparelho digestivo & $0,97(3)$ & $0,48(1)$ & $1,98(2)$ & \\
\hline & Radiologia & $0,97(3)$ & $1,45(3)$ & $0(0)$ & \\
\hline & Gastroenterologia & $0,32(1)$ & $0(0)$ & $0,99(1)$ & \\
\hline & Hematologia & $0,32(1)$ & $0(0)$ & $0,99(1)$ & \\
\hline & Med. da família e comunidade & $0,32(1)$ & $0,48(1)$ & $0(0)$ & \\
\hline & Medicina de emergência & $0,32(1)$ & $0(0)$ & $0,99(1)$ & \\
\hline & Nefrologia & $0,32(1)$ & $0(0)$ & $0,99(1)$ & \\
\hline & Neurologia & $0,32(1)$ & $0,48(1)$ & $0(0)$ & \\
\hline & Neuropediatria & $0,32(1)$ & $0,48(1)$ & $0(0)$ & \\
\hline & Tanatologia & $0,32(1)$ & $0,48(1)$ & $0(0)$ & \\
\hline & Urologia & $0,32(1)$ & $0,48(1)$ & $0(0)$ & \\
\hline & Cirurgia do trauma & $0,32(1)$ & $0,48(1)$ & $0(0)$ & \\
\hline & Não especificado & $46,43(143)$ & $49,76(103)$ & $39,60(40)$ & \\
\hline
\end{tabular}

Os valores apresentam as frequências relativa e absoluta para as perguntas avaliadas. Para a variável qualitativa "generalista", utilizou-se o teste exato de Fisher, e, para a variável qualitativa "especialista", adotou-se o teste do qui-quadrado de Pearson. A diferença estatística entre os grupos pré-internato e internato foi considerada significativa quando ${ }^{*} p<0,05$ e ${ }^{* *} p<0,001$.

Fonte: Elaborada pelos autores. 
Sobre a forma de exercer a profissão, a maioria (51,30\%) manifestou desejo de trabalharem emprego público ou particular. Entre os estudantes do pré-internato, 59,9\% desejavam atuar em emprego público ou particular. Já no ciclo do internato, $64,36 \%$ desejavam atuar como profissionais autônomos.

Dentre as dificuldades que esperavam encontrar como médicos, a maioria (57,7\%) considerou a "concorrência" (Tabela 4). Essa variável apresentou $\mathrm{n}$ de 0,0848 maior pela opção de assinalar mais de uma resposta.

Os estudantes foram solicitados a se manifestar sobre alguns aspectos do exercício da medicina classificando como "muito importante", "pouco importante" ou "nada importante". Destacou-se a classificação "muito importante" assinalada em "medicina como meio para ser útil ou ajudar pessoas" (98,38\%), "meio para adquirir conhecimento científico" indicado $(83,12 \%)$ e "meio para adquirir conhecimento humanístico" $(82,14 \%)$ (Tabela 5).

Sobre os sentimentos experimentados como estudante do curso de Medicina, os acadêmicos quantificaram oito aspectos em "muito importante", "pouco importante" ou "nada importante" (Tabela 6). Os seguintes aspectos receberam a classificação "muito importante": "nível de ansiedade" (80,52\%), "nível de estresse" (79,55\%), "sensação de sobrecarga" (73,38\%), "insegurança sobre o futuro" (57,14\%), "problemas financeiros" (40,58\%) e "preocupação com o excesso de escolas de Medicina" (53,90\%). Houve diferença significativa

Tabela 4. Como desejam exercer e dificuldades esperadas na profissão.

\begin{tabular}{|c|c|c|c|c|c|}
\hline \multirow{3}{*}{$\begin{array}{l}\text { Perguntas relacionadas ao } \\
\text { futuro depois de formados }\end{array}$} & \multirow{3}{*}{ Respostas } & Total & Pré-internato & Internato & \multirow{3}{*}{$\mathbf{P}$} \\
\hline & & $\%(n)$ & $\%(n)$ & $\%(n)$ & \\
\hline & & $100(308)$ & $67,21(207)$ & $32,79(101)$ & \\
\hline \multirow[t]{2}{*}{ Como deseja exercer a profissão } & Profissional liberal & $4,55(14)$ & $2,90(6)$ & $7,92(8)$ & 0,0759 \\
\hline & Profissional autônomo & $55,52(171)$ & $51,21(106)$ & $64,36(65)$ & * \\
\hline \multirow{3}{*}{ Principais dificuldades esperadas } & Salário & $11,50(41)$ & $8,90(21)$ & $16,70(20)$ & \multirow{3}{*}{0,0848} \\
\hline & Desemprego & $13,20(47)$ & $12,30(29)$ & $15,0(18)$ & \\
\hline & Outras & $17,50(62)$ & $17,0(40)$ & $18,30(22)$ & \\
\hline
\end{tabular}

Os valores apresentam as frequências relativa e absoluta para as perguntas avaliadas. Para as variáveis qualitativas "emprego público ou particular", "profissional liberal" e "profissional autônomo", utilizou-se o teste exato de Fisher, e, para a variável qualitativa "principais dificuldades esperadas", adotou-se o teste do qui-quadrado de Pearson. A diferença estatística entre os grupos pré-internato e internato foi considerada significativa quando ${ }^{*} p<0,05$ e ${ }^{* *} p<0,001$.

Fonte: Elaborada pelos autores.

Tabela 5. Importância da medicina no exercício da profissão.

\begin{tabular}{|c|c|c|c|c|c|}
\hline \multirow{3}{*}{$\begin{array}{c}\text { Perguntas relacionadas ao exercício } \\
\text { da profissão médica }\end{array}$} & \multirow{3}{*}{ Respostas } & Total & Pré-internato & Internato & \multirow{3}{*}{$\mathbf{P}$} \\
\hline & & $\%(n)$ & $\%(n)$ & $\%(n)$ & \\
\hline & & $100(308)$ & $67,21(207)$ & $32,79(101)$ & \\
\hline \multirow{2}{*}{ Como meio para ajudar as pessoas } & Pouco & $1,62(5)$ & $0,97(2)$ & $2,97(3)$ & \multirow{2}{*}{0,3352} \\
\hline & Muito & $98,38(303)$ & 99,03 (205) & $97,03(98)$ & \\
\hline \multirow{3}{*}{ Estabilidade financeira } & Pouco & $23,05(71)$ & $27,05(56)$ & $14,85(15)$ & \multirow{3}{*}{ * } \\
\hline & Muito & $76,30(235)$ & $71,98(149)$ & $85,15(86)$ & \\
\hline & Nada & $0,65(2)$ & $0,97(2)$ & $0(0)$ & \\
\hline \multirow{4}{*}{ Status social } & Pouco & $47,40(146)$ & $45,41(94)$ & $51,49(52)$ & \multirow{4}{*}{0,2417} \\
\hline & Muito & $2,92(9)$ & $1,93(4)$ & $4,95(5)$ & \\
\hline & Nada & $49,03(151)$ & $52,17(108)$ & $42,57(43)$ & \\
\hline & Não especificado & $0,65(2)$ & $0,48(1)$ & $0,99(1)$ & \\
\hline
\end{tabular}


Tabela 5. (Continuação) Importância da medicina no exercício da profissão.

\begin{tabular}{|c|c|c|c|c|c|}
\hline \multirow{3}{*}{$\begin{array}{l}\text { Perguntas relacionadas ao exercício } \\
\text { da profissão médica }\end{array}$} & \multirow{3}{*}{ Respostas } & Total & Pré-internato & Internato & \multirow{3}{*}{$\mathbf{P}$} \\
\hline & & $\%(n)$ & $\%(n)$ & $\%(n)$ & \\
\hline & & $100(308)$ & $67,21(207)$ & $32,79(101)$ & \\
\hline \multirow{2}{*}{ Aquisição de conhecimento científico } & Muito & $83,12(256)$ & $82,13(170)$ & $85,15(86)$ & \multirow{2}{*}{0,3715} \\
\hline & Nada & $0,32(1)$ & $0,48(1)$ & $0(0)$ & \\
\hline \multirow{3}{*}{$\begin{array}{l}\text { Aquisição de conhecimento } \\
\text { humanístico }\end{array}$} & Pouco & $16,88(52)$ & $15,46(32)$ & $19,80(20)$ & \multirow{3}{*}{0,6344} \\
\hline & Muito & $82,14(253)$ & $83,57(173)$ & $79,21(80)$ & \\
\hline & Nada & $0,97(3)$ & $0,97(2)$ & $0,99(1)$ & \\
\hline Oportunidade de lidar com pessoas & Pouco & $17,86(55)$ & $14,49(30)$ & $24,75(25)$ & * \\
\hline
\end{tabular}

Os valores apresentam as frequências relativa e absoluta para as perguntas avaliadas. Para a variável qualitativa "como meio para ajudar as pessoas", utilizou-se o teste Exato de Fisher, e, para as demais variáveis qualitativas, adotou-se o teste do qui-quadrado de Pearson. A diferença estatística entre os grupos pré-internato e internato foi considerada significativa quando ${ }^{*} p<0,05$.

Fonte: Elaborada pelos autores.

Tabela 6. Sentimentos vividos durante o curso de Medicina.

\begin{tabular}{|c|c|c|c|c|c|}
\hline \multirow{3}{*}{$\begin{array}{c}\text { Perguntas relacionadas aos } \\
\text { atuais sentimentos vividos } \\
\text { durante o curso }\end{array}$} & \multirow{3}{*}{ Respostas } & Total & Pré-internato & Internato & \multirow{3}{*}{$p$} \\
\hline & & $\%(n)$ & $\%(n)$ & $\%(n)$ & \\
\hline & & $100(308)$ & $67,21(207)$ & $32,79(101)$ & \\
\hline \multirow[b]{2}{*}{ Nível de ansiedade } & Muito & $80,52(248)$ & $77,78(161)$ & $86,14(87)$ & \multirow[b]{2}{*}{0,3494} \\
\hline & Nada & $2,92(9)$ & $3,38(7)$ & $1,98(2)$ & \\
\hline \multirow{4}{*}{ Nível de estresse } & Pouco & $18,51(57)$ & $15,94(33)$ & $23,76(24)$ & \multirow{4}{*}{0,3271} \\
\hline & Muito & $79,55(245)$ & $81,64(169)$ & $75,25(76)$ & \\
\hline & Nada & $1,62(5)$ & $1,93(4)$ & $0,99(1)$ & \\
\hline & Não especificado & $0,32(1)$ & $0,48(1)$ & $0(0)$ & \\
\hline Nível de insatisfação & Não especificado & $0,65(2)$ & $0,97(2)$ & $0(0)$ & 0,0583 \\
\hline \multirow{4}{*}{ Sensação de sobrecarga } & Pouco & $24,35(75)$ & $24,64(51)$ & $23,76(24)$ & \multirow{4}{*}{0,7696} \\
\hline & Muito & $73,38(226)$ & $72,95(151)$ & $74,26(75)$ & \\
\hline & Nada & $1,62(5)$ & $1,45(3)$ & $1,98(2)$ & \\
\hline & Não especificado & $0,65(2)$ & $0,97(2)$ & $0(0)$ & \\
\hline \multirow{3}{*}{$\begin{array}{c}\text { Sensação de insegurança sobre } \\
\text { o futuro }\end{array}$} & Pouco & $35,71(110)$ & $39,61(82)$ & $27,72(28)$ & \multirow{3}{*}{ * } \\
\hline & Muito & $57,14(176)$ & $51,69(107)$ & $68,32(69)$ & \\
\hline & Nada & $6,82(21)$ & $8,21(17)$ & $3,96(4)$ & \\
\hline
\end{tabular}


Table 6. (Continuation) Feelings experienced during the medical course.

\begin{tabular}{|c|c|c|c|c|c|}
\hline \multirow{4}{*}{ Problemas financeiros } & Pouco & $50,0(154)$ & $46,38(96)$ & $57,43(58)$ & \multirow{4}{*}{0,2697} \\
\hline & Muito & $40,58(125)$ & $43,96(91)$ & $33,66(34)$ & \\
\hline & Nada & $9,09(28)$ & $9,18(19)$ & $8,91(9)$ & \\
\hline & Não especificado & $0,32(1)$ & $0,48(1)$ & $0(0)$ & \\
\hline \multirow{4}{*}{$\begin{array}{l}\text { Dúvidas sobre a qualidade do } \\
\text { ensino }\end{array}$} & Pouco & $44,16(136)$ & $42,03(87)$ & $48,51(49)$ & \multirow{4}{*}{0,5008} \\
\hline & Muito & $41,23(127)$ & $41,55(86)$ & $40,59(41)$ & \\
\hline & Nada & $14,29(44)$ & $15,94(33)$ & $10,89(11)$ & \\
\hline & Não especificado & $0,32(1)$ & $0,48(1)$ & $0(0)$ & \\
\hline \multirow{4}{*}{$\begin{array}{l}\text { Preocupação com o excesso de } \\
\text { escolas de Medicina }\end{array}$} & Pouco & $35,71(110)$ & $32,85(68)$ & $41,58(42)$ & \multirow{4}{*}{0,0572} \\
\hline & Muito & $53,90(166)$ & $53,62(111)$ & $54,46(55)$ & \\
\hline & Nada & $10,06(31)$ & $13,04(27)$ & $3,96(4)$ & \\
\hline & Não especificado & $0,32(1)$ & $0,48(1)$ & $0(0)$ & \\
\hline
\end{tabular}

Os valores apresentam as frequências relativa e absoluta para as perguntas avaliadas. A diferença estatística entre os grupos pré-internato e internato foi considerada significativa quando ${ }^{*} p<0,05$.

Fonte: Elaborada pelos autores.

entre as respostas dos grupos em relação à "insegurança sobre o futuro" por conta do maior nível de preocupação dos acadêmicos do internato $(p<0,05)$.

\section{DISCUSSÃO}

A tendência a um maior número de mulheres no curso de Medicina confirmou-se na presente pesquisa (63,3\%), concordando com o resultado de outros dois trabalhos em que, respectivamente, $\mathbf{7 4 , 4 \%}$ e $53 \%$ dos participantes eram mulheres ${ }^{10,11}$. Os resultados encontrados seguem tendência mundial, uma vez que, a partir de 1970, as faculdades passaram a ter maior participação de mulheres, aspecto que aumentou gradativamente nas duas décadas seguintes e mais rapidamente na primeira década do século $\mathrm{XXI}^{12}$.

Em relação à principal razão que levou os participantes a escolher a medicina como profissão, obteve-se como resultado mais relevante o "altruísmo" $(71,75 \%)$, seguido da "estabilidade financeira" (59,4\%) e "realização pessoal" (59\%), conforme mostra a Tabela 1, dados que estão parcialmente de acordo com outra pesquisa em que o fator determinante para a escolha foi "ajudar e servir pessoas" ou "trabalhar com pessoas", seguido de "empregabilidade" e "bons salários". A vocação para a medicina ou afinidade com a profissão obteve menor preferência (54,2\%), assim como ocorreu em outra pesquisa em que os autores encontraram índice semelhante para a resposta "identificação com a profissão" (53,4\%), opção que abrangia tanto vocação como realização pessoal ${ }^{13}$.

Quando comparadas as repostas dos estudantes do préinternato e internato, houve, de um lado, diminuição significativa da opção pelo "altruísmo". De outro lado, a preocupação com "estabilidade financeira" elevou-se, assemelhando-se a um estudo realizado em 2011, no qual "empregabilidade", "inserção no mercado de trabalho" e "possibilidade de bons salários" foram justificativas frequentes para a escolha da medicina como profissão8.

Assim sendo, os estudantes ingressam na graduação em Medicina com a concepção principal de altruísmo, entretanto, com o conhecimento dos problemas do mercado de trabalho, despertam para a atualidade médica brasileira, e isso reduz a expectativa em relação a um possível retorno salarial generoso e a preocupação para com o outro. No entanto, o princípio da beneficência, que representa em parte o altruísmo, foi incorporado ao exercício da medicina desde seus primórdios por meio do Juramento de Hipócrates, e, desde o século passado, tornou-se o Princípio Fundamental I do Código de Ética Médica brasileiro $^{10,14}$. Por isso, embora a realidade da profissão possa desmotivar o altruísmo, essa qualidade é inerente à profissão médica e essencial para proporcionar maior benefício aos pacientes, devendo ser conservada.

Participantes de uma escola de Medicina elegeram humanismo, afinidade e status social como os três principais motivadores da escolha da profissão, evidenciando que os estudantes, além do altruísmo e vocação, podem estar fascinados pelo retorno financeiro e pela relevância social da profissão ${ }^{15}$. Na presente pesquisa, os participantes manifestaram preocupação quanto ao futuro salário em índice que variou de $8,9 \%$ no pré-internato a $16,7 \%$ no internato (Tabela 2), mostrando que a proximidade de exercer a profissão contribui para a melhor percepção do aspecto econômico. Essa realidade ficou evidente em editorial publicado pelo Conselho Regional 
de Medicina do Estado de São Paulo (Cremesp), em que foi salientada a questão dos salários aviltantes para médicos de serviços públicos, os quais se refletem desfavoravelmente no setor privado, tendência que ocorre em desconsideração às quase dez mil horas/aula durante a graduação, sem levar em conta o período pré-vestibular e a especialização ${ }^{16}$.

Uma preocupação bastante citada por ambos os grupos foi com o desemprego (13,2\% no pré-internato e 15\% no internato), corroborando outro estudo em que o excesso de escolas médicas no Brasil e as projeções indicando que, em quatro décadas, o país terá cerca de 1,5 milhão de profissionais médicos foram motivos de apreensão dos estudantes ${ }^{8}$. Ao excesso de escolas médicas, associa-se a concorrência que advirá dos milhares de estudantes que cursam Medicina em países vizinhos e buscarão incorporarse ao mercado de trabalho nacional.

$\mathrm{Na}$ escolha das especialidades (Tabela 3), transpareceu o aumento da preferência por ginecologia e obstetrícia, pediatria, cirurgia geral, psiquiatria e oncologia. A preferência pode variar segundo o sexo de estudantes, conforme apontou uma pesquisa em que as mulheres preferiram cursos como ginecologia e obstetrícia, pediatria e cuidados paliativos, inferindo-se que seja por serem portadoras de maior empatia, habilidade em ouvir e afeto, qualidades comuns à sua natureza materna que as levaram a ser menos denunciadas em Conselho de Medicina ${ }^{17}$. Na presente pesquisa, embora seja uma das mais denunciadas por erro médico ${ }^{18}$, também houve desproporcional preferência pela especialidade ginecologia e obstetrícia por parte das mulheres, mas nenhum participante escolheu cuidados paliativos. Parece não haver dúvidas de que a carreira profissional e a construção familiar são levadas em conta no momento da escolha, preocupação manifestada por $37,99 \%$ dos participantes que almejavam conciliar carreira e vida pessoal após formados.

Quase todos consideraram muito importante seu nível de ansiedade (80,52\%) e estresse (79,55\%). O curso de Medicina requer do aluno intensa dedicação e disponibilidade de tempo a fim de realizar o sonho de se tornar médico. Em pesquisa realizada com acadêmicos de um curso de Medicina, constatouse que, no decorrer da formação, estão presentes momentos estressantes que são potencialmente prejudiciais ao componente psicológico ${ }^{4}$. Isso também ficou evidente em outro estudo no qual $78 \%$ dos estudantes argumentaram que a medicina é estressante, com carga de trabalho excessiva e cumprimento de longas jornadas, acarretando menor disponibilidade de tempo para a família e o lazer ${ }^{19}$. Além disso, o fato de os estudantes raramente buscarem ajuda para seus problemas tanto resulta em prejuízos pessoais e profissionais como influi negativamente na escolha da especialidade pretendida e no futuro enfrentamento de erros cometidos durante a atuação ${ }^{20}$.
Outro resultado de destaque encontrado na presente pesquisa foi o menor desejo de acadêmicos do internato em seguir a carreira como médicos generalistas, não especialistas (Tabela 4). Esse fato também foi observado em estudo realizado na Faculdade de Medicina da Universidade Federal de Minas Gerais (UFMG) em que somente $15,4 \%$ dos mais de 700 estudantes entrevistados gostariam de trabalhar na atenção básica à saúde, e, dentre os que consideraram tornar-se médicos generalistas, nenhum era da última fase do curso ${ }^{17}$. Esse aspecto pode ser justificado pelo resultado de outra pesquisa em que $73,6 \%$ dos acadêmicos acreditavam haver menor remuneração dos médicos generalistas e $89,3 \%$ menor prestígio social ${ }^{21}$.

Segundo dados da Demografia médica no Brasil 2018, de todos os registros médicos ativos no país no ano anterior, $62,5 \%$ apresentavam um ou mais títulos de especialista, obtendose uma razão de 1,67 especialista para cada generalista ${ }^{22}$. A maioria das faculdades permanece com currículo centrado nas especialidades, aspecto que se associa à precariedade da estrutura dos serviços do Serviço Único de Saúde (SUS) em que atuam os generalistas, contribuindo para a desmotivação ${ }^{23}$. Assim, ao levarem em conta as condições menos favoráveis, o baixo salário e a instabilidade do sistema público de saúde, a maioria dos estudantes prefere alternativas de trabalho que não incluem essa opção ${ }^{13}$. Por isso, passar na residência e obter qualificação profissional alcançou índice elevado na preferência dos participantes da presente pesquisa.

A medicina é uma profissão prestada em benefício da saúde do ser humano, devendo ser desempenhada sem distinções e com o maior empenho possível ${ }^{14}$. Nesse aspecto, ficou evidente que, para quase todos, a medicina é um meio de ajudar as pessoas (Tabela 5). Contudo, o medo de cometer erros e da desvalorização da profissão e o nível de responsabilidade foram exclamativos em ambos os grupos (Tabela 2), porquanto a possibilidade de ser denunciado é real, conforme ocorreu com 613 médicos que foram a julgamento pelo Conselho Regional de Medicina do Estado de Santa Catarina (CRM-SC) no período de 2004 a $2009^{18}$.

\section{CONCLUSÕES}

As principais razões que levaram os acadêmicos a escolher a medicina como profissão foram o altruísmo e a estabilidade financeira. Contudo, quando comparadas as respostas dos estudantes do início e final do curso, houve diminuição da preferência pelo altruísmo durante o internato, e estima-se que isso se deva ao maior conhecimento da realidade do exercício profissional por esse grupo. No entanto, a percepção do exercício da medicina como meio para ajudar pessoas manteve-se elevada em ambos os grupos.

Em relação à perspectiva laboral, transpareceu o receio da limitação do mercado de trabalho e desvalorização da 
profissão. A abertura indiscriminada de escolas médicas pode estar sensibilizando o acadêmico a se preocupar com a própria carreira, visto que a maioria considerou muito importante esse aspecto.

Quase a totalidade dos participantes, sobretudo do internato, manifestou o desejo de fazer especialização, preferência que se justifica pelas limitações estruturais, técnicas e salariais do setor público que constitui a principal oportunidade de emprego para o médico generalista, não especialista. $\mathrm{O}$ aumento da ansiedade e insegurança que ocorre nas fases finais do curso condiz com o receio de não conseguir um emprego e de não ser um bom profissional.

Em conclusão, os estudantes apresentam uma visão mais idealizada da medicina nas fases iniciais, porém, entre os discentes do final do curso, houve uma diminuição dos ideais humanísticos que motivaram a escolha da profissão e um aumento da insegurança em relação ao mercado de trabalho, ao salário e à conquista do sucesso profissional.

\section{CONTRIBUIÇÃO DOS AUTORES}

Eduardo Delatorre Kamijo e Maria Victória Schweder de Lima participaram do delineamento da pesquisa, da coleta e análise dos dados, e da redação do artigo. Ana Paula Pereira participou do delineamento da pesquisa, da análise dos dados e da revisão final do artigo. Elcio Luiz Bonamigo participou como pesquisador e orientador em todas as etapas do estudo, incluindo a redação e a revisão final do artigo.

\section{CONFLITO DE INTERESSES}

Declaramos não haver conflito de interesses.

\section{FINANCIAMENTO}

Declaramos não haver financiamento.

\section{REFERÊNCIAS}

1. Ramos-Cerqueira ATA, Lima MCP. A formação da identidade do médico: implicações para o ensino de graduação em Medicina. Interface. 2002;6(11):107-16. doi: 10.1590/S1414-32832002000200008

2. Diretório das Escolas de Medicina. Painel de indicadores. 2020 [access in 16 feb 2021]. Available from: https://produtos.direm.org/painel

3. Millan LR, Azevedo RS, Rossi E, De Marco OLN, Millan MPB, Arruda PCV. What is behind a student's choice for becoming a doctor? Clinics. 2005;60(2):143-50. doi: 10.1590/S1807-59322005000200011.

4. Santos FS, Maia CRC, Faedo FC, Gomes GPC, Nunes ME, Oliveira MVM. Estresse em estudantes de cursos preparatórios e de graduação em Medicina. Rev Bras Educ Med. 2017;41(2):194-200. doi: 10.1590/1981-52712015v41n2rb20150047.

5. Aragao JCS, Rossi HR, Casiraghi B. A jornada do acadêmico de Medicina: um modelo simbólico da formação médica. Rev Bras Educ Med. 2018;42(1):40-6. doi: 10.1590/1981-52712018v42n1rb20170037.
6. Miranda IMM, Tavares HHF, Silva HRS, Braga MS, Santos RO, Guerra HS. Qualidade de vida e graduação em Medicina. Rev Bras Educ Med. 2020;44(3):1-8. doi: 10.1590/S0100-55022020000300206.

7. Kam SXL, Toledo ALS, Pacheco CC, Souza GFB, Santana VLM, Bonfá-Araujo, $B$ et al. Estresse em estudantes ao longo da graduação médica. Rev Bras Educ Med. 2020;43(1):247-53. doi: 10.1590/S0100-55022019000500246.

8. Ribeiro MMF, Leal SS, Diamantino FC, Bianchi HA. A opção pela medicina e os planos em relação ao futuro profissional de estudantes de uma faculdade pública brasileira. Rev Bras Educ Med. 2011;35(3):405-11. doi: 10.1590/S0100-55022011000300015.

9. Barreto MA, Reis CN, Miranda IB, Jardim LCR, Teixeira MP. Ser médico: o imaginário coletivo de estudantes de medicina acerca da profissão de médico. Cadernos Unifoa. 2017;4(11):73-6.

10. Monteiro MFA, Barbosa JMO, Carteado EMF, Ferreira MAD, André AM Opção pelo curso de Medicina em Angola: o caso da Universidade Agostinho Neto. Rev Bras Educ Med. 2010;34(3):346-54. doi: 10.1590/ S0100-55022010000300003.

11. Moreira SNT, Silva CAN, Tertulino FF, Tertulino FMF, Vilar MJP, Azevedo GD. Processo de significação de estudantes do curso de medicina diante da escolha profissional e das experiências vividas no cotidiano acadêmico. Rev Bras Educ Med. 2006;30(2):14-9. doi: 10.1590/S010055022006000200003.

12. Avila RC. Formação das mulheres nas escolas de medicina. Rev Bras Educ Med. 2014; 38(1):142-9 [access in 18 sep 2019]. Available from: https:// www.scielo.br/pdf/rbem/v38n1/19.pdf.

13. Ferreira RA, Peret Filho LA, Goulart EMA, Valadão MMA. O estudante de medicina da Universidade Federal de Minas Gerais: perfil e tendências. Rev Assoc Med Bras. 2000;46(3):224-31. doi: 10.1590/S010442302000000300007.

14. Conselho Federal de Medicina. Resolução $n^{\circ} 2.217$, de 27 de setembro de 2018. Brasília; CFM; 2018.

15. Trindade LMDF, Vieira MJ. Curso de Medicina: motivações e expectativas de estudantes. Rev Bras Educ Med. 2009;33(4):542-54. doi: 10.1590/S01005502200900040000 .

16. Conselho Regional de Medicina do Estado de São Paulo. Salários dos médicos no serviço público: ultraje. São Paulo: Cremesp; 2008 [access in 16 may 2019]. Available from: https://www.cremesp.org. $\mathrm{br} /$ ?siteAcao $=$ Jornal\&id $=946$.

17. Lempp $\mathrm{H}$, Seale $\mathrm{C}$. Medical students' perceptions in relation to ethnicity and gender: a qualitative study. BMC Med Educ. 2006;17(6):1-7. doi: 10.1186/1472-6920-6-17.

18. Koeche LG, Cenci I, Bortoluzzi MC, Bonamigo EL. Prevalência de erro médico entre as especialidades médicas nos processos julgados pelo Conselho Regional de Medicina do Estado de Santa Catarina. ACM Arq Catarin Med. 2013;45(4):45-53.

19. Saad SMA, Fatima SS, Faruqi AA. Students' views regarding selecting medicine as a profession. J Pak Med Assoc. 2011;61(8):832-6.

20. Maia HAAS, Assunção ACS, Silva CS, Santos JLP, Menezes CJJ, Bessa Jr J. Prevalência de sintomas depressivos em estudantes de Medicina com currículo de aprendizagem baseada em problemas. Rev Bras Educ Med. 2020;44(3):1-7. doi: 10.1590/\$0100-55022020000300219.

21. Chehuen Neto JA, Sirimarco MT, Cândido TC, Ulhoa CM, Reis BP, Lima VM. Formação médica generalista: percepção do profissional e do estudante. HU Rev. 2014;40(1-2):13-23 [access in 5 oct 2021]. Available from: https:// periodicos.uff.br/index.php/hurevista/article/view/2095.

22. Scheffer $M$, coordenador. Demografia médica no Brasil 2018. São Paulo: FMUSP, CFM, Cremesp; 2018.

23. Silva LO, Melo IB, Teixeira LAS. Interface entre oferta de vagas de residência médica, demanda por médicos especialistas e mercado de trabalho. Rev Bras Educ Med. 2020;43(1):119-26. doi: 10.1590/S010055022020000300219 . 